\title{
Assessment of natural variation in the first pore domain of the tomato HKT1;2 transporter and characterization of mutated versions of SIHKT1;2 expressed in Xenopus laevis oocytes and via complementation of the salt sensitive athkt1;1 mutant
}

\author{
Pedro M. F. Almeida ${ }^{1}{ }^{*}$, Gert-Jan de Boer ${ }^{2}$ and Albertus H. de Boer ${ }^{1}$ \\ ${ }^{1}$ Department of Structural Biology, Faculty Earth and Life Sciences, Vrije Universiteit Amsterdam, Amsterdam, Netherlands \\ ${ }^{2}$ R\&D Department, Enza Zaden, Enkhuizen, Netherlands
}

\author{
Edited by: \\ Andreas P. M. Weber, \\ Heinrich-Heine-Universität, Germany \\ Reviewed by: \\ Vadim Volkov, London Metropolitan \\ University, UK \\ Alexandre Boscari, Institut National \\ de la Recherche Agronomique, \\ France \\ *Correspondence: \\ Pedro M. F. Almeida, Department of \\ Structural Biology, Faculty Earth and \\ Life Sciences, Vrije Universiteit \\ Amsterdam, De Boelelaan 1085, \\ 1081 HV Amsterdam, Netherlands \\ e-mail: p.m.fidalgodealmeida@vu.nl
}

Single Nucleotide Polymorphisms (SNPs) within the coding sequence of HKT transporters are important for the functioning of these transporters in several plant species. To unravel the functioning of HKT transporters analysis of natural variation and multiple site-directed mutations studies are crucial. Also the in vivo functioning of HKT proteins, via complementation studies performed with athkt1;1 plants, could provide essential information about these transporters. In this work, we analyzed the natural variation present in the first pore domain of the HKT1;2 coding sequence of 93 different tomato accessions, which revealed that this region was conserved among all accessions analyzed. Analysis of mutations introduced in the first pore domain of the SIHKT1;2 gene showed, when heterologous expressed in Xenopus laevis oocytes, that the replacement of S70 by a $\mathrm{G}$ allowed SIHKT2;1 to transport $\mathrm{K}^{+}$, but also caused a large reduction in both $\mathrm{Na}^{+}$ and $\mathrm{K}^{+}$mediated currents. The study of the transport characteristics of SIHKT1;2 revealed that $\mathrm{Na}^{+}$-transport by the tomato SIHKT1;2 protein was inhibited by the presence of $\mathrm{K}^{+}$ at the outside of the membrane. GUS expression under the AtHKT1;1 promoter gave blue staining in the vascular system of transgenic Arabidopsis. athkt1;1 mutant plants transformed with AtHKT1;1, SIHKT1;2, AtHKT1;1S68G, and SIHKT1;2S70G indicated that both AtHKT1;1 and SIHKT1;2 were able to restore the accumulation of $\mathrm{K}^{+}$in the shoot, although the low accumulation of $\mathrm{Na}^{+}$as shown by WT plants was only partially restored. The inhibition of $\mathrm{Na}^{+}$transport by $\mathrm{K}^{+}$, shown by the SIHKT1;2 transporter in oocytes (and not by AtHKT1;1), was not reflected in $\mathrm{Na}^{+}$accumulation in the plants transformed with SIHKT1;2. Both AtHKT1;1-S68G and SIHKT1;2-S70G were not able to restore the phenotype of athkt1;1 mutant plants.

Keywords: natural variation, tomato, HKT1 genes, mutation, pore domain

\section{INTRODUCTION}

Salinity stress negatively affects crop yield (Munns and Tester, 2008). In order to sustain the growing human population it is necessary to increase the salt tolerance of crop plants, as the human population is growing faster than the area of agricultural land (FAO, 2009). To tolerate salinity plants rely on three different mechanisms: osmotic tolerance, ionic tolerance and $\mathrm{Na}^{+}$exclusion from the shoots (Munns and Tester, 2008). $\mathrm{Na}^{+}$exclusion from the shoots is the most studied and best understood mechanism, therefore, it is a promising candidate for an approach of genetic modification to enhance plant salt tolerance (Plett et al., 2010).

HKT transporters are often studied with regard to $\mathrm{Na}^{+}$exclusion from the shoots. HKT transporters belong to a superfamily of transporters including bacterial KtrBs transporters (Tholema et al., 1999) and yeast TRKs transporters (Rodriguez-Navarro, 2000). The HKT gene family is divided in two classes based on their gene structure and in the presence of either a glycine $(\mathrm{G})$ or a serine (S) residue in the first pore domain of the transporter (Maser et al., 2002). Members of class 1 have an $S$ at this position, whereas members of class 2, with the exception of OsHKT2;1, have a $\mathrm{G}$ at this position (Platten et al., 2006). HKT transporters are implicated in $\mathrm{Na}^{+}$transport in wheat (Davenport et al., 2005; James et al., 2006; Byrt et al., 2007; Munns et al., 2012), rice (Ren et al., 2005; Horie et al., 2007; Jabnoune et al., 2009) and Arabidopsis (Uozumi et al., 2000; Berthomieu et al., 2003; Rus et al., 2004; Sunarpi et al., 2005; Moller et al., 2009). Class I HKT transporters are low affinity transporters with specificity for $\mathrm{Na}^{+}$(Munns and Tester, 2008). Some of these members are located at the plasma membrane of root stele cells, in particular 
in the xylem parenchyma cells (XPC). They function in retrieving $\mathrm{Na}^{+}$from the xylem sap, and prevent $\mathrm{Na}^{+}$from reaching the shoots and damaging photosynthetic cells. The number of class I HKT members varies between mono- and dicotyledonous plants (Garciadeblas et al., 2003; Ren et al., 2005; Huang et al., 2006; Jabnoune et al., 2009). When first characterized, athkt1;1 and wild type (WT) seedlings showed no difference in root and shoot growth after growing 6 days in a medium with $(150 \mathrm{mM})$ or without $\mathrm{NaCl}$ (Rus et al., 2004). However, on the long term medium supplemented with $75 \mathrm{mM} \mathrm{NaCl}$ reduced the shoot growth and increased tip senescence of mature leaves of athkt1;1 mutants (Rus et al., 2004). Due to the higher $\mathrm{Na}^{+}$accumulation in the shoots, athkt1;1 mutant plants display $\mathrm{Na}^{+}$sensitivity, showing the role of HKT transporters in preventing $\mathrm{Na}^{+}$from reaching the shoots (Rus et al., 2001, 2004; Berthomieu et al., 2003; Sunarpi et al., 2005).

The discovery of genetic polymorphisms in HKT genes (Diatloff et al., 1998; Rubio et al., 1999; Ren et al., 2005; Cotsaftis et al., 2012) underlying the adaptation to salinity stress brought new insights in the understanding of the functions of genes involved in the adaptation mechanisms. Information on genetic polymorphisms in HKT genes will provide tools for the development of crops more tolerant to salinity stress. Within the same genus, the diversity of phenotypes across environmental gradients of stress can indicate the suitability for selection, and the study of the genotypes responsible for those phenotypes might lead to the discovery of genetic polymorphisms responsible for these adaptive responses (Baxter et al., 2010). Analysis of OsHKT1;5 in two rice cultivars differing in their salinity tolerance showed that both the cellular location and expression patterns of OsHKT1;5 were identical. Nevertheless, differences in the coding region producing four amino acid substitutions were linked to the functional variation of these two alleles (Ren et al., 2005). Recently, it was concluded that the V395L substitution present in Nona Bokra could directly affect the $\mathrm{Na}^{+}$transport rates and that it was responsible for the tolerant and sensitive behavior of Nona Bokra and Koshihikari, respectively (Cotsaftis et al., 2012).

Besides natural variation, studies of single (Diatloff et al., 1998; Maser et al., 2002) or multiple (Kato et al., 2007) site-directed mutations of HKT transporters (Diatloff et al., 1998; Maser et al., 2002; Kato et al., 2007) are also crucial to understand how these transporters function. The replacement of the $S$ by a $G$ in the first pore domain of AtHKT1;1 changed AtHKT1;1 from a Na${ }^{+}$ uniporter to a $\mathrm{Na}^{+} / \mathrm{K}^{+}$symporter (Maser et al., 2002).

Data on the role of HKT transporters is often generated from heterologous expression of HKT transporters in, mainly, Xenopus laevis oocytes (Schachtman and Schroeder, 1994; Fairbairn et al., 2000; Uozumi et al., 2000; Horie et al., 2001; Liu et al., 2001; Golldack et al., 2002; Berthomieu et al., 2003; Su et al., 2003; Ren et al., 2005; Jabnoune et al., 2009; Lan et al., 2010; Yao et al., 2010; Horie et al., 2011) and, to a lesser extent, in Saccharomyces cerevisiae cells (Horie et al., 2001, 2011; Golldack et al., 2002; Garciadeblas et al., 2003; Su et al., 2003; Takahashi et al., 2007; Ali et al., 2012). However, in a report by Haro et al. (2005) a very important question was addressed: are results obtained for HKT transporters when expressed in heterologous systems of physiological importance in planta? Or, are heterologous expression systems too artificial to have physiological meaning in planta? We performed several experiments to investigate if there is natural variation present in the first pore domain of HKT1;2 in tomato and how the replacement of the $S$ residue by a $G$ residue in the first pore domain in HKT1;2 of tomato affects the function of the transporter when expressed in Xenopus laevis oocytes. Moreover, we investigated if the results obtained with Xenopus oocytes could be replicated in planta.

In this study we analyzed the presence of natural variation in the first pore domain of HKT1;2 of 93 tomato accessions. We also replaced the $S$ residue of the first pore domain of SlHKT1;2 with a $G$ residue, and we analyzed the effect of this mutation via expression of these mutated genes in Xenopus laevis oocytes. We generated stable lines of Arabidopsis thaliana athkt1;1 plants expressing each of these constructs, and we characterized their biomass production, shoot water-content and ion content after 2 weeks of salt treatment.

\section{MATERIALS AND METHODS \\ PLANT MATERIAL AND GROWTH CONDITIONS}

Genomic DNA used in this experiment was extracted from 93 different tomato accessions (Supplementary File Table 1). Tomato seeds were surface sterilized by soaking in $1 \%(\mathrm{~V} / \mathrm{V})$ commercial sodium hypochlorite solution for $15 \mathrm{~min}$ and rinsed three times with sterile water. After sterilization, seeds were sown in rock wool plugs soaked with half-strength Hoagland solution (one seed per rock wool plug). Plugs were covered with dry vermiculite to avoid dehydration. A randomized design consisting of three biological replicates and 93 different accessions was used. Each biological replica consisted of a pool of seven to 10 plants. On alternate days plants were irrigated with half-strength Hoagland solution. Plants were kept in a climate chamber under a $14 / 10 \mathrm{~h}$ photoperiod and a $20 / 18^{\circ} \mathrm{C}$ day/night temperature. Plants were harvested after 5 weeks. Seeds of homozygous athkt1;1 mutant (Columbia- 0 ) were obtained from the NASC stock center (N6531) and were sown along with WT Arabidopsis thaliana Col-0. Plants were grown on a mix of sand and peat $(1: 1)$ at $24^{\circ} \mathrm{C}$ in a $16 \mathrm{~h}$ light $/ 8 \mathrm{~h}$ dark cycle in a greenhouse. Plants were watered every 2 days. Selected transgenic lines (See the section "Cloning of HKT genes and generation of Arabidopsis transgenic lines" for a full description of how these lines were generated) were grown under the same conditions. Four-week old transgenic lines (T2 lines) were treated with $100 \mathrm{mM} \mathrm{NaCl}$ every 2 days during 2 weeks before harvesting of shoot material.

\section{GENOMIC DNA EXTRACTION}

For the extraction of genomic DNA approximately $25 \mathrm{mg}$ of dried root material of each tomato accession was weighed and inserted in a 96 deep well plate. Samples were freeze dried for 1 week using a freeze dryer (Christ Alpha 1-4 LD plus, Germany). For the extraction of DNA a Nucleospin 96 Plant II kit (MachereyNagel, Germany) was used and the manufacturer's protocol was followed. The quality of the gDNA was checked on a $1.5 \%$ agarose gel. The concentration of the gDNA was calculated using the Quanti-iTTM PicoGREEN dsDNA assay kit (Invitrogen). gDNA was diluted in TE buffer $\mathrm{pH} 8.0$ (10 mM Tris and $1 \mathrm{mM}$ EDTA) and stored at $4^{\circ} \mathrm{C}$. 


\section{PRIMER AND PROBE DESIGN AND ANALYSIS OF NATURAL VARIATION}

Primers and probes (Supplementary File Table 2) were designed to have a Tm between 60 and $67^{\circ} \mathrm{C}$ with DNASIS MAX v3.0 software. Pairs of primers were designed to flank the SNP under study. The size of amplicons was designed to be smaller than 150 nucleotides. Unlabeled probes were blocked at the $3^{\prime}$ end to prevent extension in PCR reactions and designed to anneal over an SNP of interest. Reactions were performed in 384-well plates. $20 \mathrm{ng}$ of gDNA per sample were used to study natural variation in tomato HKT1;2 nucleotides of different cultivars. Per reaction, $3.21 \mu \mathrm{l}$ of MilliQ, $0.05 \mu \mathrm{l}$ of FW primer $(10 \mu \mathrm{mol} / \mu \mathrm{l})$ and $0.25 \mu \mathrm{l} \mathrm{RV}$ primer $(10 \mu \mathrm{mol} / \mu \mathrm{l}), 0.04 \mu \mathrm{l}$ of PAL $(5 \mathrm{U} / \mu \mathrm{l})$ (KAPA Biosystems, Boston, USA), $0.25 \mu \mathrm{l}$ LC Green (Idaho Technology, Salt Lake City, USA), $0.20 \mu \mathrm{l}$ dNTP $(5 \mathrm{mM})$ and $1 \mu \mathrm{l}$ PAL buffer (KAPA Biosystems, Boston, USA) supplemented with $12.5 \mathrm{mM}$ $\mathrm{MgCl}_{2}$, were added. The amplification reaction started with a denaturation step of $95^{\circ} \mathrm{C}$ for $10 \mathrm{~min}$ and continued with 14 cycles of $95^{\circ} \mathrm{C}$ for $15 \mathrm{sec}$ and $60^{\circ} \mathrm{C}$ for $4 \mathrm{~min}$. Samples were cooled to room temperature and a first melting curve analysis was performed to assess the quality of the amplification. Samples were again cooled down to room temperature and $2 \mu \mathrm{l}(10 \mathrm{pmol} / \mu \mathrm{l})$ of probe was added. Samples were heated up to $96^{\circ} \mathrm{C}$ for $3 \mathrm{~min}$ and cooled down to room temperature before analyzing the melting curve of the probe. The mix of PCR products and probes was heated up to $96^{\circ} \mathrm{C}$ for $3 \mathrm{~min}$ and then cooled down to room temperature to allow hetero-duplex formation. Thereafter, the mix of PCR products and probes was re-heated to $95^{\circ} \mathrm{C}$ at $0.3^{\circ} \mathrm{C} / \mathrm{s}$. Data were acquired between 50 and $95^{\circ} \mathrm{C}$. Data acquisition was made with a Light Scanner HR384 (Idaho Technology Inc. Salt Lake City, USA). High Resolution Melting (HRM) curve analysis was performed using the "Unlabeled Probes" module in the "genotyping" mode of the software. This mode involves negative filter, normalization and grouping. SlHKT1;2 coding sequence isolated from S. lycopersicum Arbasson F1 was used as a reference. Amplicons that showed a melting curve different from the reference melting-curve were selected, amplified and sequenced. Sequencing of the amplicons was performed at Macrogen Europe Laboratories, Amsterdam, The Netherlands.

\section{PLASMID CONSTRUCTION}

Site-directed mutagenesis of SlHKT1;2 was conducted using overlap extension PCR. All primers used to make mutated HKT1 genes are listed in (Supplementary File Table 3). pGEMHESphI+SlHKT1;2 and pGEM-HESphI+AtHKT1;1 (see Almeida et al., 2014 for a full description of how these constructs were obtained) were used as template and the corresponding mutated gene cloned into the $\mathrm{BamHI}$ and $\mathrm{XbaI}$ restriction sites and SphI and BamHI restriction sites of an empty pGEM-HESphI vector for tomato and Arabidopsis HKT1, respectively. The constructs obtained were called pGEM-HESphI+AtHKT1;1-S68G and $p G E M-H E S p h I+S l H K T 1 ; 2-S 70 G$. All PCR-derived DNAfragments were confirmed by sequencing.

\section{HETEROLOGOUS EXPRESSION OF HKT1 GENES IN XENOPUS LAEVIS OOCYTES}

Preparation of template DNA, in vitro transcription and capping of mRNA and Two-electrode voltage clamping of Xenopus oocytes was performed according to Almeida et al. (2014). All measurements were performed at least 3 times on oocytes isolated from two different batches.

\section{CLONING OF HKT GENES, GENERATION OF ARABIDOPSIS TRANSGENIC LINES AND GUS STAINING}

The $p G E M-H E$ vector used in this work was modified to contain the SphI restriction site. This restriction site was introduced between the BamHI and XbaI restriction sites present in the vector. This was made by designing two complementary primers containing the SphI restriction site flanked by both BamHI and $\mathrm{XbaI}$ restriction sites, and introducing this piece of DNA between the BamHI and XbaI restriction sites of $p G E M-H E$ vector. The $p G E M-H E$ vector containing this new restriction site was selected and used in this work. To be able to distinguish the original $p G E M-H E$ and $p G E M-H E$ containing the SphI restriction site, we called our vector $p G E M-H E S p h I$.

pGEM-HESphI+AtHKT1;1, pGEM-HESphI+AtHKT1;1S68G, pGEM-HESphI+SlHKT1;2, and pGEM-HESphI+ SlHKT1;2-S70G were used as template in the amplification of the HKT genes flanked by the attB Gateway (Invitrogen) recombination sites. The AtHKT1;1, AtHKT1;1-S68G, SlHKT1;2, and SlHKT1;2-S70G genes were cloned into the pDONR221 $P 5-P 2$ vector (Invitrogen) and were named p5-2AtHKT1;1, p5-2AtHKT1;1-S68G, p5-2SlHKT1;2, and p5-2SlHKT1;2-S70G, respectively. A $5 \mathrm{~kb}$ DNA fragment upstream of the $A T G$ start codon of the AtHKT1;1 gene containing the promoter region, the tandem repeat and the small RNA target region (Baek et al., 2011) was cloned into pDONR221 P1-P5 and pDONR221 $P 1-P 2$ (Invitrogen) and the resultant constructs were named p1-5AtHKT1;1prom, p1-2AtHKT1;1prom, respectively. Cloning of DNA fragments into pDONR221 (Invitrogen) vectors was performed by BP reactions (Invitrogen). Cloning of either AtHKT or SlHKT genes into pHGW (Karimi et al., 2002) under the AtHKT1;1 promoter was performed by LR reactions (Invitrogen). In this way, $p H G W+A t H K T 1 ; 1$ prom $+A t H K T 1 ; 1$, pHGW+AtHKT1;1prom +AtHKT1;1-S68G, pHGW+AtHKT1; 1 prom + SlHKT1;2, and pHGW+AtHKT1;1prom+SlHKT1;2S70G constructs were created. p1-2AtHKT1;1prom was incubated with pKGWFS7 (Karimi et al., 2002) in an LR reaction (Invitrogen) to create the $p K G W F S 7+A t H K T 1 ; 1$ prom construct. All constructs were sequenced prior to the transformation of Arabidopsis plants. All primers used are listed (Supplementary File Table 4). All constructs were introduced into Agrobacterium tumefaciens strain GV3101pMP90, including the $p H G W$ empty vector, and transformed into 3-week old athkt1-1 mutant plants, except pKGWFS7+AtHKT1;1prom and the $p K G W F S 7$ empty vector, which were transformed into 3-week old Arabidopsis WT plants. Plant transformation was performed by the flower dipping method (Clough and Bent, 1998). athkt1;1 mutant plants (N6531) (Rus et al., 2001) were transformed with $p H G W+A t H K T 1 ; 1$ prom $+A t H K T 1 ; 1$ or pHGW+AtHKT1;1prom $+A t H K T 1 ; 1-S 68 G$ or $p H G W+A t H K T 1$; 1prom + SlHKT1;2 or pHGW+AtHKT1;1prom+SlHKT1;2S70G. Arabidopsis WT plants were transformed with pKGWFS7+AtHKT1;1prom or pKGWFS7 empty vector. Four weeks after transformation, seeds were harvested and surface 
sterilized. Surface sterilization was performed by washing seeds during $1 \mathrm{~min}$ with a $80 \%$ ethanol solution with $0.1 \%$ Tween-20, followed by a $20 \mathrm{~min}$ washing step with $1 \%$ commercial bleach, and three washing steps with sterile MilliQ. MilliQ was then replaced by warm half-strength Murashige and Skoog (MS) medium supplemented with $1 \%$ sucrose, $0.8 \%$ agar and $10 \mathrm{mg} / \mathrm{L}$ hygromycin or $50 \mathrm{mg} / \mathrm{L}$ kanamycin. Seeds were placed in round plates containing solid MS medium with the same composition. Seedlings showing hygromycin or kanamycin resistance were selected and transferred to pots containing a mix of soil and peat (1:1). They were grown for 4 weeks under the growing conditions described above, after which the seeds were harvested. T2 seeds were tested for antibiotic resistance and used to either investigate their growth response under different $\mathrm{NaCl}$ concentrations or for GUS staining. Histochemical assays of GUS activity (biological replicates $n=3$ ) were conducted using samples that were stained according to Jefferson et al. (1987). Seven days old plantlets (plantlets $n=7$ ) were incubated at $37^{\circ} \mathrm{C}$ for $6 \mathrm{~h}$ in the staining solution containing $0.5 \mathrm{mM} \mathrm{X}$-Gluc (5-bromo-4-chloro-3-indolyl-b-D-glucoronide).

\section{ANALYSIS OF TRANSCRIPTS LEVELS}

Tissue specific expression of all HKT genes was tested by extracting total RNA from roots and leaves of three-week old WT and transformed Arabidopsis lines. RNA from transformed plants was extracted and immediately frozen in liquid nitrogen. RNA extraction was performed using the Innuprep Plant RNA kit (Westburg, the Netherlands). First strand cDNA was synthesized using one microgram of total RNA, random hexamers and SuperScript II Reverse Transcriptase (Invitrogen Life Technologies). First strand cDNA was used as a template for quantitative real-time PCR (qRT-PCR). Samples were measured from each transformed plant using a sequence detector system (7300 Real Time PCR System from Applied Biosystems). Primers used to test the expression of AtHKT1;1 and AtHKT1;1-S68G according to (Jha et al., 2010). Primers used to test the expression of SlHKT1;2 and SlHKT1;2S70G according to Asins et al. (2012). $\beta$-Actin transcript levels were used as an internal standard. The mean normalized expression was calculated according to Livak and Schmittgen (2001).

\section{STATISTICAL ANALYSES OF DATA}

Two-way ANOVA was used to assess the effect of salt treatment on HKT1 gene expression, fresh weight, $\mathrm{Na}^{+}$and $\mathrm{K}^{+}$accumulation. Normality and homogeneity assumptions were checked. Gene expression data were rank-transformed if these assumptions were violated. If ANOVA was significant, a post hoc test (Tukey's test) was used to evaluate differences among treatment and plant lines. All tests were performed using SPSS 17.0.

\section{SEQUENCE DATA}

The SlHKT1;2 sequence used in this study has the EMBL/GenBank accession number HG530660 (Almeida et al., 2014).

\section{RESULTS}

\section{SLHKT1;2 S7O IS CONSERVED THROUGHOUT SOLANUM ACCESSIONS}

From all 93 tomato accessions tested only three (PI 126435, LA 2931 and LA 1401) showed different melting curves (Figure 1A).

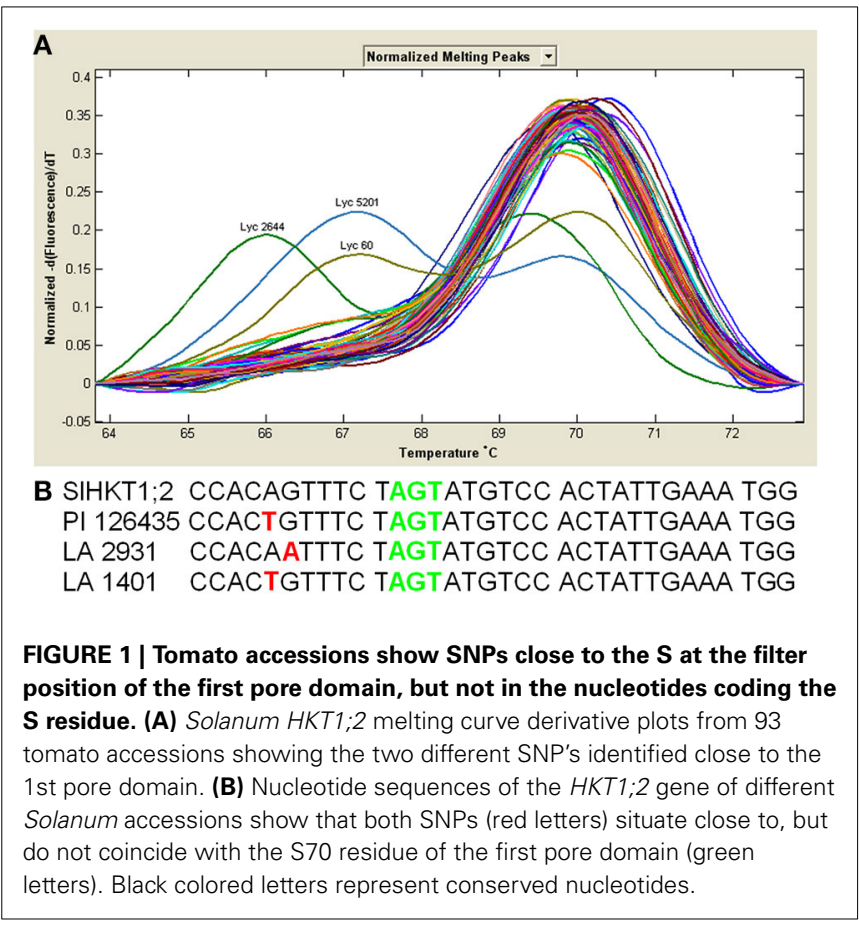

To identify whether the SNPs responsible for these different melting curves were located at our target region, we amplified and sequenced the region of the first pore domain of these three accessions (Figure 1B). Sequencing results revealed that each of these three accessions have a single SNP (PI 126435 and LA 1401 have the same SNP and LA 2931 has a different SNP), although none of them in the position of interest. The SNP in the accession LA 2931 resulted in an amino acid change from valine (V) to isoleucine (I); however, both amino acids are hydrophobic. In the case of accessions PI 126435 and LA 1401 the SNP did not result in any amino acid change, as both ACA and ACT code for a threonine (T) residue.

\section{SITE-DIRECTED MUTAGENESIS OF S70 TO G OF SLHKT1;2}

To test the hypothesis that S70 of the tomato SIHKT1;2 protein is crucial for the $\mathrm{Na}^{+}$selectivity we replaced $\mathrm{S} 70$ by a G (SlHKT1;2-S70G). cRNA of SlHKT1;2-S70G was injected in Xenopus laevis oocytes. After 2 days of incubation, currents produced in the presence of $\mathrm{Na}^{+}$and $\mathrm{K}^{+}$ions were recorded in oocytes expressing both WT and mutated HKT1 transporters from Arabidopsis thaliana and Solanum lycopersicum $(n=3)$ (Figure 2). AtHKT1;1-S68G expressing oocytes (Maser et al., 2002) were used as a positive control of SlHKT1;2-S70G. Currents produced by oocytes expressing either AtHKT1;1 (Figure 2A) or SlHKT1;2 (Figure 2I) increased when the oocytes were bathed in higher $\mathrm{Na}^{+}$concentrations (as seen by a more negative current). Increasing external $\mathrm{K}^{+}$concentration did not result in any change in the current levels produced by AtHKT1;1 expressing oocytes (Figure 2B). In contrast, $S l H K T 1 ; 2$ mediated inward and outward currents were sensitive to external $\mathrm{K}^{+}$concentration as both currents decreased with increasing bath $\mathrm{K}^{+}$concentration (Figure 2J). Increased concentrations of $\mathrm{K}^{+}$result in an inhibition on the transport of $\mathrm{Na}^{+}$by SIHKT1;2 but not by 


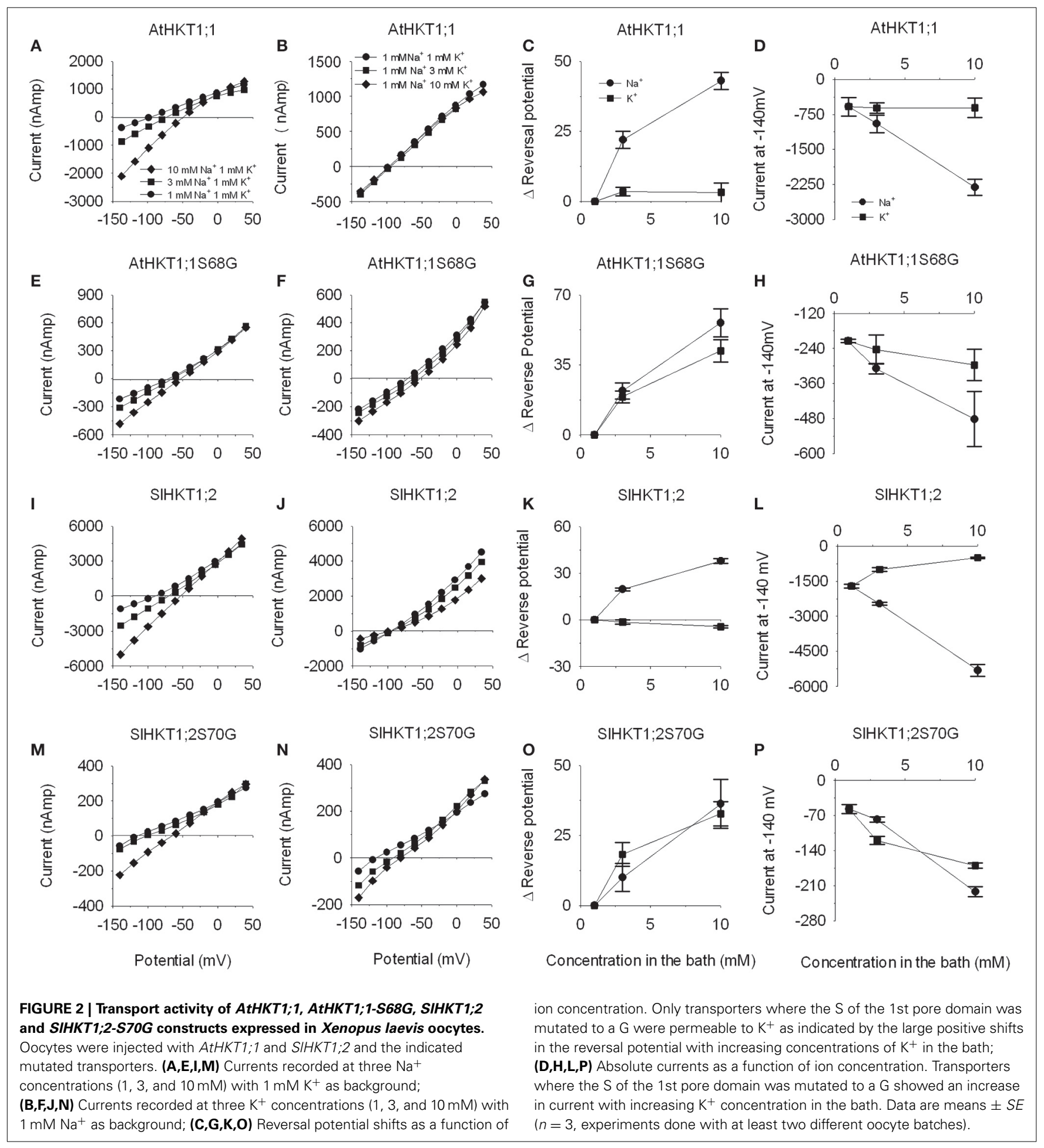

AtHKT1;1. When oocytes expressing either AtHKT1;1-S68G or SlHKT1;2-S70G were bathed with either increasing $\mathrm{Na}^{+}$concentration (Figures 2E,M) or $\mathrm{K}^{+}$concentration (Figures 2F,N) currents increased for both cations tested. For both AtHKT1;1and SlHKT1;2-mediated currents a higher $\mathrm{Na}^{+}$concentration but not a higher $\mathrm{K}^{+}$concentration resulted in positive shifts in the reversal potential (Figures $\mathbf{2} \mathbf{C}, \mathbf{K}$ ), which is indicative of $\mathrm{Na}^{+}$permeation. Reversal potentials obtained with oocytes expressing either AtHKT1;1-S68G (Figure 2G) or SlHKT1;2-S70G (Figure 2O) showed positive shifts when both $\mathrm{Na}^{+}$concentration or $\mathrm{K}^{+}$concentration increased, indicating that the presence of a $G$ residue at the filter position of the first pore domain allows the transport of both $\mathrm{Na}^{+}$and $\mathrm{K}^{+}$ions. Figures 2D,H,L,P show the currents recorded at $-140 \mathrm{mV}$ for AtHKT1;1, AtHKT1;1-S68G, 


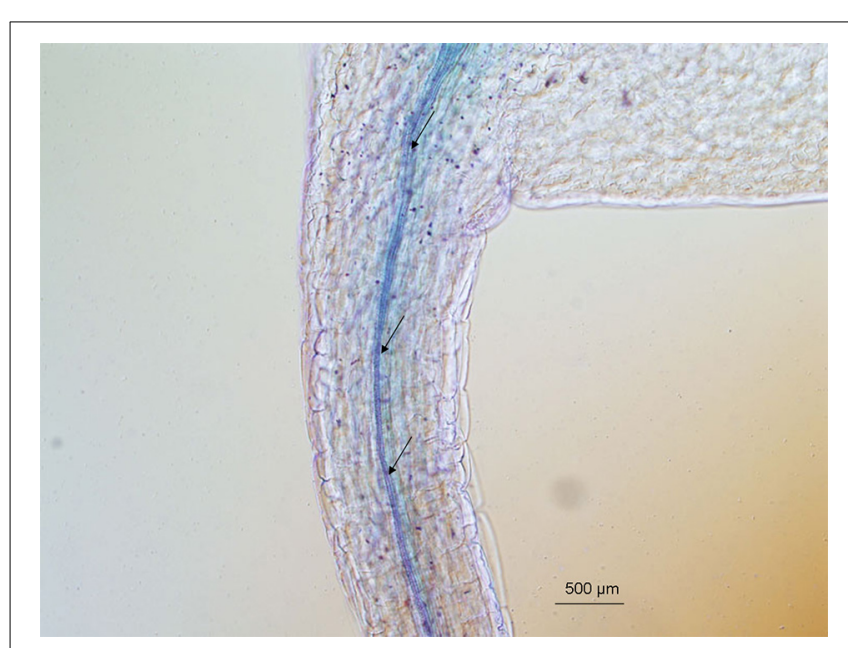

FIGURE 3 | Detection of GUS activity in the vascular system of transgenic Arabidopsis thaliana plants. Arabidopsis plants expressed the GUS gene under the control of the AtHKT1;1 promoter were grown. Strong blue GUS staining was detected in the vicinity of the xylem and phloem in leaves. Arrows point to the xylem vessels. Biological replicates, $n=3$; plantlets, $n=7$.

SlHKT1;2 and SlHKT1;2-S70G, respectively. These results show that the $\mathrm{Na}^{+}$-mediated current of SIHKT1;2 is reduced by increased concentrations of $\mathrm{K}^{+}$in the bath. The presence of $\mathrm{K}^{+}$ ions affects the transport of $\mathrm{Na}^{+}$by SlHKT1;2. This effect is not observed with AtHKT1;1.

\section{SELECTION AND MOLECULAR ANALYSIS OF T2 PLANTS AND GUS EXPRESSION IN A. THALIANA UNDER THE A. THALIANA HKT1;1}

HKT1 gene expression was analyzed in T2 Arabidopsis plantlets carrying the SlHKT1;2 or AtHKT1;1, SlHKT1;2-S70G, or AtHKT1;1-S68G genes (data not shown). All genes were expressed under the AtHKT1;1 endogenous promoter. All lines tested showed HKT1 expression levels higher than the athkt1;1 (N6531) mutant plants. Expression of AtHKT1;1 was not detected in athkt1;1 mutant plants. The $5 \mathrm{~kb}$ AtHKT1;1 promoter fragment was able to drive the expression of GUS in the vascular tissues of transformed Arabidopsis WT plants (Figure 3). GUS activity driven by AtHKT1;1 promoter was only observed in the vascular tissues of leaves of transgenic Arabidopsis plants (Figure 3).

\section{ANALYSIS OF FW, $\mathrm{Na}^{+}$, AND $\mathrm{K}^{+}$ACCUMULATION OF TRANSGENIC ARABIDOPSIS LINES}

There was a marked difference in $\mathrm{K}^{+}$-sensitivity of $\mathrm{Na}^{+}$-transport mediated by the AtHKT1;1 and SIHKT1;2 proteins, as analyzed in Xenopus laevis oocytes. To see whether this difference was reflected in $\mathrm{Na}^{+} / \mathrm{K}^{+}$homeostasis in planta, we expressed the AtHKT1;1 and SlHKT1;2 genes driven by a $5 \mathrm{~kb}$ long AtHKT1;1 promoter in the athkt1;1 mutant plants and studied how they complemented the mutant phenotype. Since the analysis of SlHKT1;2-S70G and AtHKT1;1-S68G in Xenopus oocytes showed interesting effects on transport activity and ion selectivity we transformed athkt1;1 plants with SlHKT1;2-S70G and AtHKT1$S 68 G$, and tested the functioning of these mutated genes in planta

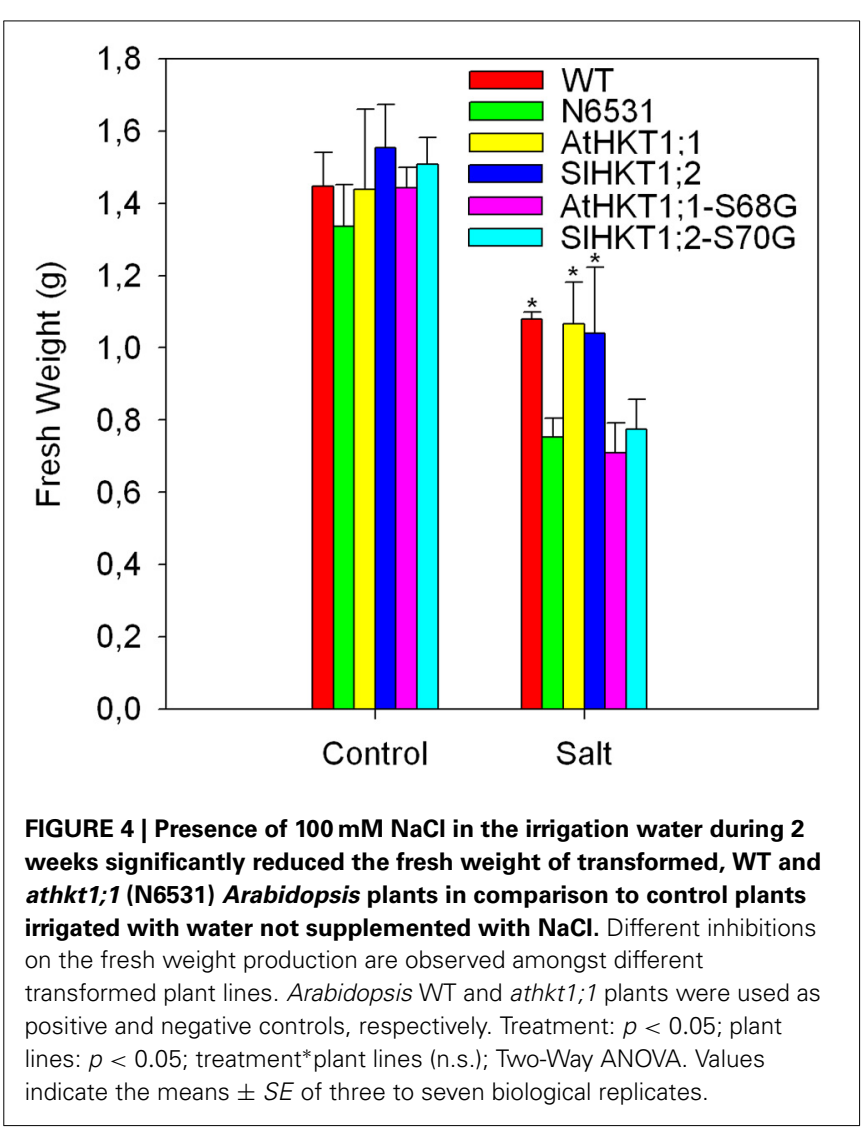

during salinity stress. Figure 4 shows the effect of salinity treatment on the leaf fresh weight of WT plants, athkt1;1 mutants and transformed plants. athkt1;1-mutant plants were more sensitive to salt than the WT plants. Both the AtHKT1;1 and the SlHKT1;2 gene were able to complement the athkt1;1 mutant growth-phenotype on salt (Figure 4). Plants transformed with SlHKT1;2-S70G or AtHKT1-S68G were just as sensitive to salt as the athkt1;1-mutant plants. The analysis of the relative water content of the leaves did not show statistically significant differences between control and salt treated plants (data not shown) nor within transformed lines. The effect of the athkt1;1 mutation on $\mathrm{Na}^{+}$and $\mathrm{K}^{+}$homeostasis was pronounced (Figure 5). The athkt1;1 plants accumulated almost four-fold more $\mathrm{Na}^{+}$and two-fold less $\mathrm{K}^{+}$, resulting in an eight-fold higher $\mathrm{Na}^{+} / \mathrm{K}^{+}$-ratio in the shoot of the mutant plants as compared to the WT plants. The transgenic lines that complemented the athkt1;1 growthphenotype (AtHKT1;1 and SlHKT1;2) showed effects on ion accumulation: the athkt1;1 $\mathrm{K}^{+}$-phenotype during salt stress (i.e., strong reduction in $\mathrm{K}^{+}$accumulation) was completely restored, but $\mathrm{Na}^{+}$accumulation in the leaves of these transgenic lines was still significantly higher than that of the WT plants.

\section{DISCUSSION}

Several studies have shown that naturally occurring SNPs in genes involved in $\mathrm{Na}^{+}$and $\mathrm{K}^{+}$homeostasis can have a dramatic effect on the salinity tolerance of several plant species. This is the case for HKT transporters (Rubio et al., 1995; Ren et al., 2005; Baxter et al., 2010; Ali et al., 2012; Cotsaftis et al., 2012). Due to the 


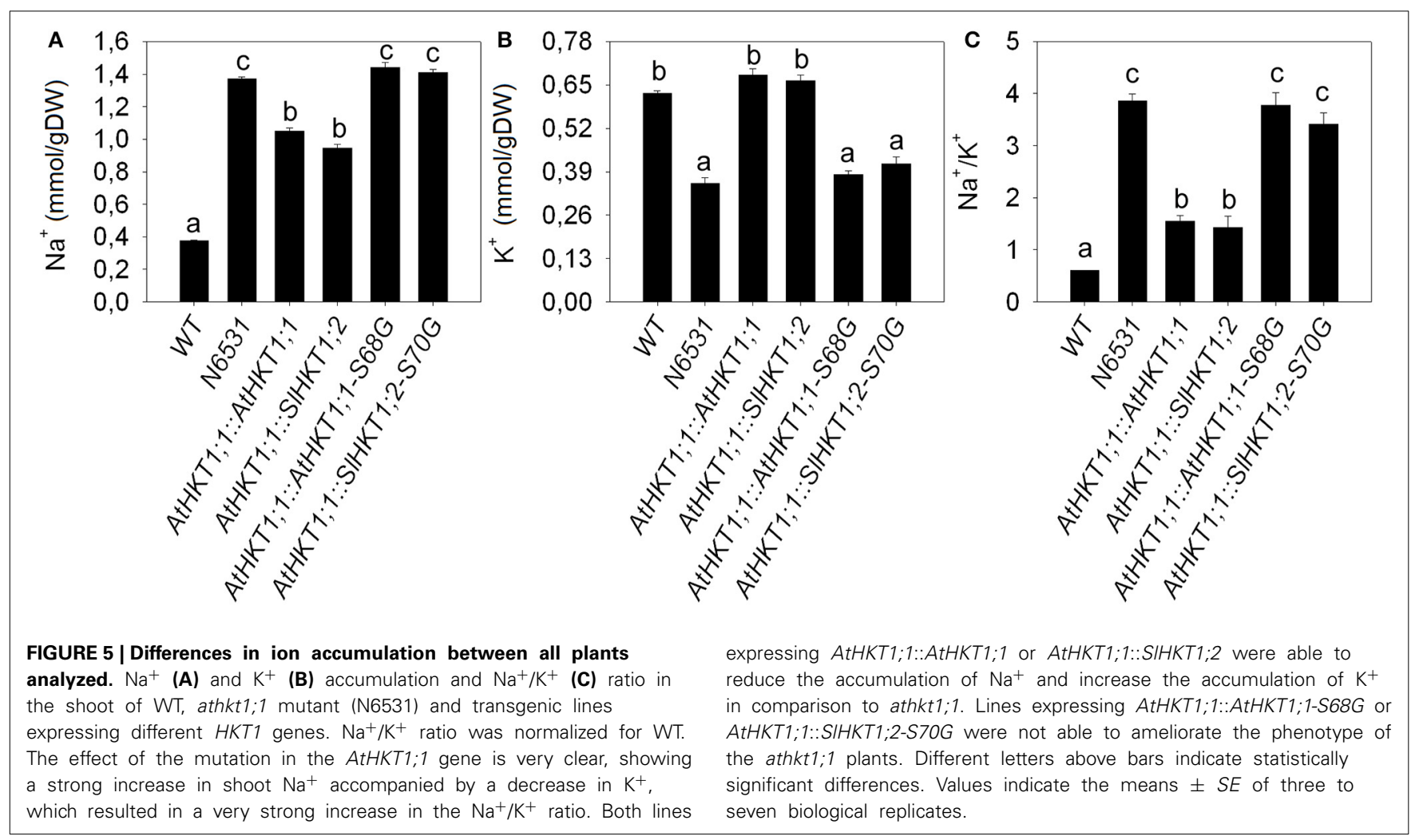

effects of SNPs on the transport properties of HKT proteins, and consequently the effect of these SNPs on the salinity tolerance of the plants, we decided to study the presence/absence of SNPs in the sequence of the first pore domain of the HKT1;2 gene of several tomato accessions (Figure 1). The first pore domain in all accessions studied showed an S, which is naturally occurring in AtHKT1;1, implying that if during evolution the amino acid residue that we studied would have changed, these changes were not in favor of salinity tolerance of the tomato plants and, therefore, did not persist (Diatloff et al., 1998).

The analysis of the properties of the heterologous expressed SlHKT1;2 showed that the transport characteristics were in accordance with the presence of an $S$ in the first pore domain of the transporter (Figure 2). This is reflected in the SlHKT1;2 transport characteristics as measured in heterologous expression, where it was shown that tomato $\mathrm{HKT} 1 ; 2$ transports $\mathrm{Na}^{+}$but not $\mathrm{K}^{+}$ (Almeida et al., 2014). However, a striking difference between the transport properties of AtHKT1;1 and SlHKT1;2 expressed in oocytes was observed when currents were measured at constant $\mathrm{Na}^{+}$in the bath $(1 \mathrm{mM})$ and increasing $\mathrm{K}^{+}(1,3$, and $10 \mathrm{mM})$. Whereas the AtHKT1;1 mediated current was virtually insensitive to higher $\mathrm{K}^{+}$, the SlHKT1;2 mediated current decreased by $60 \%$ at $10 \mathrm{mM} \mathrm{K}^{+}$(Figure 2). A similar inhibitory action of $\mathrm{K}^{+}$on HKT-mediated currents was reported for OsHKT2;1 (Jabnoune et al., 2009) and for TmHKT1;5-A (Munns et al., 2012). It was proposed that this inhibition is caused by the association of $\mathrm{K}^{+}$ to the $\mathrm{Na}^{+}$binding region within the pore region of HKT transporters (Rubio et al., 1995; Gassmann et al., 1996). This inhibition has not been observed with AtHKT1;1 (Uozumi et al., 2000) nor
OsHKT1;5 (Ren et al., 2005) in Xenopus oocytes, which indicates that the $\mathrm{K}^{+}$-sensitivity of these transporters is different from OsHKT2;1, TmHKT1;5-A and tomato SlHKT1;2. Physiologically, this $\mathrm{K}^{+}$-induced reduction of $\mathrm{Na}^{+}$-influx might mean that the tomato plants maintain a certain $\mathrm{K}^{+} / \mathrm{Na}^{+}$-homeostasis in the transpiration sap. High concentrations of $\mathrm{K}^{+}$in the xylem sap might imply a reduced $\mathrm{Na}^{+}$-uptake into the XPC, as the $\mathrm{Na}^{+} / \mathrm{K}^{+}$ ratio is in favor of $\mathrm{Na}^{+}$. On the other hand, low concentrations of $\mathrm{K}^{+}$and high concentrations of $\mathrm{Na}^{+}$in the xylem sap imply an induced $\mathrm{Na}^{+}$-uptake into the XPC, as the $\mathrm{Na}^{+} / \mathrm{K}^{+}$ratio is in favor of $\mathrm{K}^{+}$in the xylem sap. In this model, the $\mathrm{Na}^{+} / \mathrm{K}^{+}$ ratio is more important than the absolute concentration of $\mathrm{Na}^{+}$ in the xylem sap in determining the $\mathrm{Na}^{+}$uptake into the XPC by HKT1 (Figure 6). Although the $S \rightarrow G$ mutation in the first pore domain of both the AtHKT1;1 and SIHKT1;2 protein had an effect on the ion selectivity of both transporters, as deduced from the shift in reversal potential at increasing external $\mathrm{K}^{+}$(Figure 2), a major difference observed was the reduction in total current transported by SIHKT1;2-S70G and AtHKT1-S68G, which was 95 and $78 \%$ respectively of that transported by the WT proteins at $10 \mathrm{mM} \mathrm{Na}^{+}$and $1 \mathrm{mM} \mathrm{K}^{+}$in the bath (Figure 2 ). The reason for this difference is unclear as the results we obtained do not match the results of the $\mathrm{Na}^{+}$currents produced by AtHKT1;1S68G reported by Maser et al. (2002). Their results did not show this reduction in total currents. Interestingly, when Maser et al. (2002) mutated AtHKT1;1 in M69L, a reduction in outward currents with increasing $\mathrm{K}^{+}$concentrations in the bath was observed, whereas in TaHKT1;2 the reverse mutation, L92M, abolished this inhibitory effect of $\mathrm{K}^{+}$on outward currents (Maser et al., 2002). 


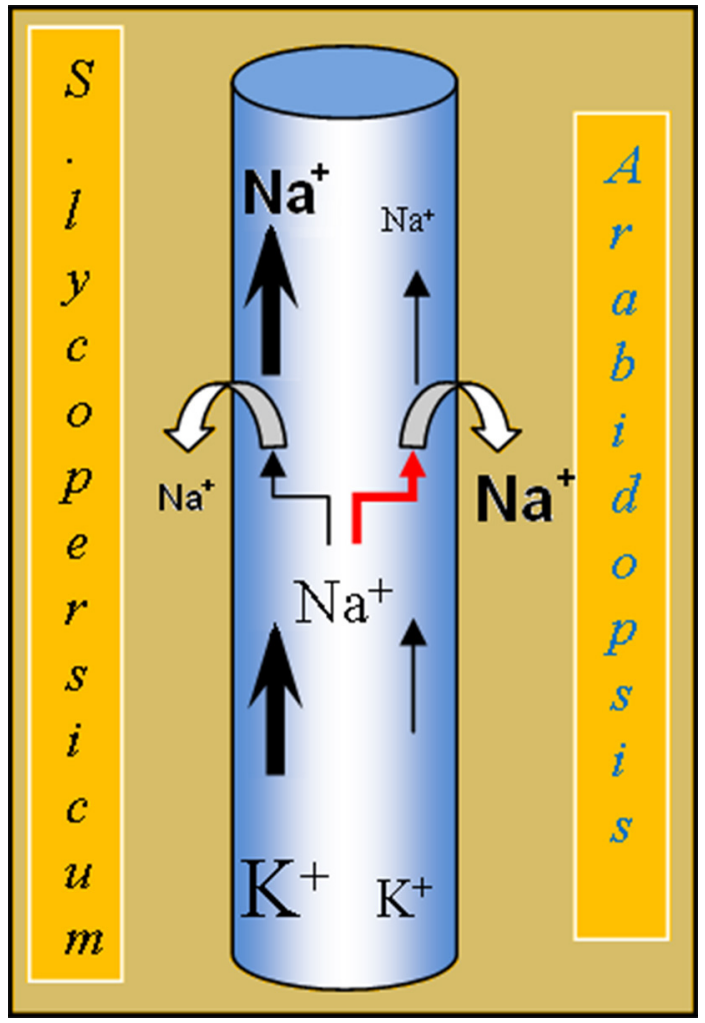

FIGURE 6 | Model depicting the difference in $\mathrm{K}^{+}$-sensitivity of SIHKT1;2 from $S$. esculentum and AtHKT1;1 from Arabidopsis. When the $\mathrm{K}^{+}$-concentration in the xylem sap is high, $\mathrm{Na}^{+}$-uptake by the SIHKT1;2 transporter is reduced. As a result the amount of $\mathrm{Na}^{+}$in the xylem stream reaching the shoot of tomato is, at least partially, controlled by SIHKT1;2, which in turn depends on the concentration of $\mathrm{K}^{+}$present in the xylem sap. In Arabidopsis, $\mathrm{Na}^{+}$-uptake in the XPCs by the AtHKT1;1 transporter is not affected by high $\mathrm{K}^{+}$.

In their work, the leucine (L) adjacent to the $\mathrm{G}$ of the pore domain seems to confer $\mathrm{K}^{+}$sensitivity to outward currents in contrast with the methionine $(\mathrm{M})$ at that same position, which abolished the effect of $\mathrm{K}^{+}$on $\mathrm{Na}^{+}$currents (Maser et al., 2002). Since in our study SlHKT1;2 has a M adjacent to the $\mathrm{G}$ of the pore domain, no sensitivity of outward currents was expected due to increasing $\mathrm{K}^{+}$concentrations in the bath. Nevertheless, in our study we observed outward currents, mediated by SIHKT1;2, sensitive to external $\mathrm{K}^{+}$. In a future study it would be interesting to mutate SlHKT1;2-M71L, to check whether the presence of a L adjacent to the $S$ of the first pore domain of SIHKT1;2 enhances or decreases this inhibitory effect of external $\mathrm{K}^{+}$on the outward $\mathrm{Na}^{+}$-currents mediated by SlHKT1;2.

\section{ATHKT1;1 AND SLHKT1;2 EXPRESSING LINES}

All plant lines studied showed a reduction in fresh weight caused by the salt treatment, and as expected the salt-induced growth reduction was stronger in the athkt1;1-mutant plants than in WT plants. With respect to growth on salt, AtHKT1;1 and SlHKT1;2 expression complemented the mutant, since the fresh weight of these lines was comparable to that of WT plants treated with salt and significantly higher than the growth of athkt1;1 plants (Figure 4). Although expression of AtHKT1;1 and SlHKT1;2 completely restored the concentration of $\mathrm{K}^{+}$in the shoot to the same level of the WT plants, $\mathrm{Na}^{+}$levels were still higher in AtHKT1;1 and SlHKT1;2-expressing lines in comparison to WT plants. Both AtHKT1;1 and SlHKT1;2 expressing lines accumulated significantly less $\mathrm{Na}^{+}$than the other transgenic lines, but significantly more $\mathrm{Na}^{+}$than the WT plants. This indicates that in these two transgenic lines both HKT1 genes do not retrieve the same amount of $\mathrm{Na}^{+}$from the xylem as WT plants. This difference in $\mathrm{Na}^{+}$accumulation in lines expressing AtHKT1;1 and SlHKT1;2 in comparison to WT plants is surprising. In this study we used the native AtHKT1;1 promoter (Baek et al., 2011) to drive the expression of all genes studied, avoiding non-native promoters as these are frequently referred to as the cause of unexpected results (Cominelli and Tonelli, 2010). The expression of GUS driven by the native AtHKT1;1 promoter (Figure 3) showed that the expression patterns were similar to previous results (Sunarpi et al., 2005) and also the level of HKT-expression in the different transgenic lines was comparable to that in WT plants.

When expressed in Xenopus laevis oocytes AtHKT1;1 and SlHKT1;2 differed in two ways: first, SlHKT1;2 but not AtHKT1;1 showed an inhibition of $\mathrm{Na}^{+}$transport by $\mathrm{K}^{+}$, and second, the total $\mathrm{Na}^{+}$-mediated current (i.e., the turn-over) measured in $S l H K T 1 ; 2$ expressing oocytes was considerably higher than the total $\mathrm{Na}^{+}$-mediated current in AtHKT1;1 expressing oocytes (Figure 2). The latter conclusion needs further testing, since it is based on the assumption that injection of the same amount of cRNA results in the same amount of protein expressed in the plasma membrane. However, it is clear that in these two transgenic lines both HKT1 transporters are involved in the retrieval of $\mathrm{Na}^{+}$ions from the xylem, as previously demonstrated for HKT1 transporters from several species (Liu et al., 2001; Laurie et al., 2002; Berthomieu et al., 2003; Garciadeblas et al., 2003; Ren et al., 2005; Horie et al., 2007; Jabnoune et al., 2009; Moller et al., 2009; Baxter et al., 2010; Plett et al., 2010; Baek et al., 2011; Mian et al., 2011; Ali et al., 2012; Munns et al., 2012).

As shown in Figure 5, mutating the athkt1;1 gene not only results in an increase in shoot $\mathrm{Na}^{+}$concentration, but also in a strong reduction in shoot $\mathrm{K}^{+}$levels. This effect on $\mathrm{K}^{+}$accumulation has been reported before (Rus et al., 2004, 2005), but a good explanation for this effect is missing thus far. Moller et al. (2009) reported that the increase in $\mathrm{K}^{+}$concentration in the shoots from plants over-expressing AtHKT1;1, was a pleiotropic effect and a consequence of the reduced $\mathrm{Na}^{+}$shoot content. Another explanation is that the uptake of $\mathrm{Na}^{+}$from the xylem into the XPCs via HKT1 results in the depolarization of the membrane potential of XPCs and activation of the depolarization activated $\mathrm{K}^{+}$-efflux channel SKOR, resulting in more $\mathrm{K}^{+}$release into the xylem (Sunarpi et al., 2005). A third explanation might be that AtHKT1;1 functionally interacts with a $\mathrm{K}^{+}$-efflux transporter in the plasma membrane of XPCs. Support for this hypothesis is found in a recently published large-scale membrane interaction screen based on a yeast mating split-ubiquitin system (mbSUS) (Membrane-based Interactome Network Database, MIND: http://cas-biodb.cas.unt.edu/project/ mind/index.php). In this screen, AtHKT1;1 was reported to 
interact with KEA3, a putative $\mathrm{K}^{+}$-efflux antiporter and member of the Proton Antiporter-2 (CPA2) family. However, this needs to be confirmed in planta in order to provide an explanation for the $\mathrm{K}^{+}$-phenotype of the athkt1;1 mutant. The absence of the AtHKT1;1 protein in the plasma membrane of XPCs of athkt1;1 plants may have a negative effect on the KEA3 antiporter, resulting in reduced root to shoot $\mathrm{K}^{+}$-transport. These hypotheses are certainly worth testing in view of the importance of $\mathrm{Na}^{+} / \mathrm{K}^{+}$-homeostasis during salinity stress.

In conclusion, the analysis of the presence of SNPs in the first pore domain of the HKT1;2 gene in 93 tomato accessions showed that this amino acid is conserved in all these accessions. The replacement of S70 by a G in SlHKT1;2 proved to be sufficient to alter the transport specificity of this transporter, as analyzed by heterologous expression in Xenopus laevis oocytes. Moreover, when expressed under the $A t H K T 1 ; 1$ native promoter, both $A t H K T 1 ; 1$ and SlHKT1;2 partially complemented the $\mathrm{Na}^{+}$ accumulation phenotype and fully complemented the $\mathrm{K}^{+}$accumulation phenotype of athkt1;1 mutant plants. AtHKT1;1-S68G and SIHKT1;2-S70G were unable to complement either the $\mathrm{Na}^{+}$ or the $\mathrm{K}^{+}$phenotype of athkt1;1 mutant plants. The transport activity of mutated HKT proteins, measured in Xenopus oocytes, was not reflected in altered $\mathrm{Na}^{+} / \mathrm{K}^{+}$-homeostasis of the athkt1;1 mutants grown under soil conditions.

\section{ACKNOWLEDGMENTS}

The authors would like to thank Enza Zaden The Netherlands for the financial support. We thank the reviewers for their valuable comments, which helped to improve this manuscript.

\section{SUPPLEMENTARY MATERIAL}

The Supplementary Material for this article can be found online at: http://www.frontiersin.org/journal/10.3389/fpls.2014. 00600/abstract

\section{REFERENCES}

Ali, Z., Park, H. C., Ali, A., Oh, D. H., Aman, R., Kropornicka, A., et al. (2012). TsHKT1;2, a HKT1 homolog from the extremophile Arabidopsis relative Thellungiella salsuginea, shows $\mathrm{K}^{+}$specificity in the presence of $\mathrm{NaCl}$. Plant Physiol. 158, 1463-1474. doi: 10.1104/pp.111.193110

Almeida, P., de Boer, G., and de Boer, A. H. (2014). Differences in shoot $\mathrm{Na}^{+}$accumulation between two tomato species are due to differences in ion affinity of HKT1;2. J. Plant Physiol. 171, 438-447. doi: 10.1016/j.jplph.2013.12.001

Asins, J. M., Villalta, I., Aly, M. M., Olias, R., Morales, P. A., Huertas, R., et al. (2012). Two closely linked tomato $H K T$ coding genes are positional candidates for the major tomato QTL involved in $\mathrm{Na}^{+} / \mathrm{K}^{+}$homeostasis. Plant Cell Environ. 36, 1171-1191. doi: 10.1111/pce.12051

Baek, D., Jiang, J. F., Chung, J. S., Wang, B. S., Chen, J. P., Xin, Z. G., et al. (2011). Regulated AtHKT1;1 gene expression by a distal enhancer element and DNA methylation in the promoter plays an important role in salt tolerance. Plant Cell Physiol. 52, 149-161. doi: 10.1093/pcp/pcq182

Baxter, I., Brazelton, J. N., Yu, D. N., Huang, Y. S., Lahner, B., Yakubova, E., et al. (2010). A coastal cline in sodium accumulation in Arabidopsis thaliana is eriven by natural variation of the sodium transporter AtHKT1;1. PLoS Genet. 6:e1001193 doi: 10.1371/journal.pgen.1001193

Berthomieu, P., Conejero, G., Nublat, A., Brackenbury, W. J., Lambert, C., Savio, C., et al. (2003). Functional analysis of AtHKT1 in Arabidopsis shows that $\mathrm{Na}^{+}$ recirculation by the phloem is crucial for salt tolerance. EMBO J. 22, 2004-2014. doi: 10.1093/emboj/cdg207

Byrt, C. S., Platten, J. D., Spielmeyer, W., James, R. A., Lagudah, E. S., Dennis, E. S., et al. (2007). HKT1;5-like cation transporters linked to $\mathrm{Na}^{+}$ exclusion loci in wheat, Nax2 and Kna1. Plant Physiol. 143, 1918-1928. doi: 10.1104/pp.106.093476

Clough, S. J., and Bent, A. F. (1998). Floral dip: a simplified method for Agrobacterium-mediated transformation of Arabidopsis thaliana. Plant J. 16, 735-743. doi: 10.1046/j.1365-313x.1998.00343.x

Cominelli, E., and Tonelli, C. (2010). Transgenic crops coping with water scarcity. N. Biotechnol. 27, 473-477. doi: 10.1016/j.nbt.2010.08.005

Cotsaftis, O., Plett, D., Shirley, N., Tester, M., and Hrmova, M. (2012). A twostaged model of $\mathrm{Na}^{+}$exclusion in rice explained by 3D modeling of HKT transporters and alternative splicing. PLOS ONE 7:e39865. doi: 10.1371/journal.pone.0039865

Davenport, R. J., James, R. A., Zakrisson-Plogander, A., Tester, M., and Munns, R. (2005). Control of sodium transport in durum wheat. Plant Physiol. 137, 807-818. doi: 10.1104/pp.104.057307

Diatloff, E., Kumar, R., and Schachtman, D. P. (1998). Site directed mutagenesis reduces the $\mathrm{Na}^{+}$affinity of $\mathrm{HKT} 1$, an $\mathrm{Na}^{+}$energized high affinity $\mathrm{K}^{+}$ transporter. FEBS Lett. 432, 31-36. doi: 10.1016/S0014-5793(98)00833-3

Fairbairn, D. J., Liu, W. H., Schachtman, D. P., Gomez-Gallego, S., Day, S. R., and Teasdale, R. D. (2000). Characterisation of two distinct HKT1-like potassium transporters from Eucalyptus camaldulensis. Plant Mol. Biol. 43, 515-525. doi: 10.1023/A:1006496402463

FAO. (2009). FAO Land and Plant Nutrition Management Service. Available online at: http://www.fao.org/ag/agl/agll/spush/.

Garciadeblas, B., Senn, M. E., Banuelos, M. A., and Rodriguez-Navarro, A. (2003). Sodium transport and HKT transporters: the rice model. Plant J. 34, 788-801. doi: 10.1046/j.1365-313X.2003.01764.x

Gassmann, W., Rubio, F., and Schroeder, J. I. (1996). Alkali cation selectivity of the wheat root high-affinity potassium transporter HKT1. Plant J. 10, 869-882. doi: 10.1046/j.1365-313X.1996.10050869.x

Golldack, D., Su, H., Quigley, F., Kamasani, U. R., Munoz-Garay, C., Balderas, E., et al. (2002). Characterization of a HKT-type transporter in rice as a general alkali cation transporter. Plant J. 31, 529-542. doi: 10.1046/j.1365313X.2002.01374.x

Haro, R., Banuelos, M. A., Senn, M. A. E., Barrero-Gil, J., and Rodriguez-Navarro, A. (2005). HKT1 mediates sodium uniport in roots. Pitfalls in the expression of HKT1 in yeast. Plant Physiol. 139, 1495-1506. doi: 10.1104/pp.105.067553

Horie, T., Brodsky, D. E., Costa, A., Kaneko, T., Lo Schiavo, F., Katsuhara, M., et al. (2011). $\mathrm{K}^{+}$transport by the OsHKT2; 4 transporter from rice with atypical $\mathrm{Na}^{+}$ transport properties and competition in permeation of $\mathrm{K}^{+}$over $\mathrm{Mg}^{2+}$ and $\mathrm{Ca}^{2+}$ ions. Plant Physiol. 156, 1493-1507. doi: 10.1104/pp.110.168047

Horie, T., Costa, A., Kim, T. H., Han, M. J., Horie, R., Leung, H. Y., et al. (2007). Rice OsHKT2; 1 transporter mediates large $\mathrm{Na}^{+}$influx components into $\mathrm{K}^{+}$-starved roots for growth. EMBO J. 26, 3003-3014. doi: 10.1038/sj.emboj. 7601732

Horie, T., Yoshida, K., Nakayama, H., Yamada, K., Oiki, S., and Shinmyo, A. (2001). Two types of HKT transporters with different properties of $\mathrm{Na}^{+}$ and $\mathrm{K}^{+}$transport in Oryza sativa. Plant J. 27, 129-138. doi: 10.1046/j.1365313x.2001.01077.x

Huang, S. B., Spielmeyer, W., Lagudah, E. S., James, R. A., Platten, J. D., Dennis, E. S., et al. (2006). A sodium transporter (HKT7) is a candidate for Nax1, a gene for salt tolerance in durum wheat. Plant Physiol. 142, 1718-1727. doi: 10.1104/pp.106.088864

Jabnoune, M., Espeout, S., Mieulet, D., Fizames, C., Verdeil, J. L., Conejero, G., et al. (2009). Diversity in expression patterns and functional properties in the rice HKT transporter family. Plant Physiol. 150, 1955-1971. doi: 10.1104/pp.109.138008

James, R. A., Davenport, R. J., and Munns, R. (2006). Physiological characterisation of two genes for $\mathrm{Na}^{+}$exclusion in durum wheat: Nax1 and Nax2. Plant Physiol. 142, 1537-1547. doi: 10.1104/pp.106.086538

Jefferson, R. A., Kavanagh, T. A., and Bevan, M. W. (1987). GUS fusion: betaglucuronidase as a sensitive and versatile gene fusion marker in higher plants. EMBO J. 20, 3901-3907.

Jha, D., Shirley, N., Tester, M., and Roy, S. J. (2010). Variation in salinity tolerance and shoot sodium accumulation in Arabidopsis ecotypes linked to differences in the natural expression levels of transporters involved in sodium transport. Plant Cell Environ. 33, 793-804. doi: 10.1111/j.1365-3040.2009.02105.x

Karimi, M., Inze, D., and Depicker, A. (2002). GATEWAY vectors for Agrobacterium-mediated plant transformation. Trends Plant Sci. 7, 193-195. doi: 10.1016/S1360-1385(02)02251-3 
Kato, N., Akai, M., Zulkifli, L., Matsuda, N., Kato, Y., Goshima, S., et al. (2007). Role of positively charged amino acids in the M2(D) transmembrane helix of Ktr/Trk/HKT type cation transporters. Channels 1, 161-171. doi: 10.4161/chan.4374

Lan, W. Z., Wang, W., Wang, S. M., Li, L. G., Buchanan, B. B., Lin, H. X., et al. (2010). A rice high-affinity potassium transporter (HKT) conceals a calciumpermeable cation channel. Proc. Natl. Acad. Sci. U.S.A. 107, 7089-7094. doi: 10.1073/pnas.1000698107

Laurie, S., Feeney, K. A., Maathuis, F. J., Heard, P. J., Brown, S. J., and Leigh, R. A. (2002). A role for HKT1 in sodium uptake by wheat roots. Plant J. 32, 139-149. doi: 10.1046/j.1365-313X.2002.01410.x

Liu, W., Fairbairn, D. J., Reid, R. J., and Schachtman, D. P. (2001). Characterization of two HKT1 homologues from Eucalyptus camaldulensis that display intrinsic osmosensing capability. Plant Physiol. 127, 283-294. doi: 10.1104/pp. 127.1.283

Livak, K. J., and Schmittgen, T. D. (2001). Analysis of relative gene expression data using real-time quantitative PCR and the 2-deltadeltaCT method. Methods 25, 402-408. doi: 10.1006/meth.2001.1262

Maser, P., Hosoo, Y., Goshima, S., Horie, T., Eckelman, B., Yamada, K., et al. (2002). Glycine residues in potassium channel-like selectivity filters determine potassium selectivity in four-loop-per-subunit HKT transporters from plants. Proc. Natl. Acad. Sci. U.S.A. 99, 6428-6433. doi: 10.1073/pnas.082123799

Mian, A., Oomen, R. J., Isayenkow, S., Sentenac, H., Maathuis, F. J., and Very, A. A. (2011). Overexpression of a $\mathrm{Na}^{+}$and $\mathrm{K}^{+}$-permeable HKT transporter in barley improves salt tolerance. Plant J. 68, 468-479. doi: 10.1111/j.1365313X.2011.04701.x

Moller, I. S., Gilliham, M., Jha, D., Mayo, G. M., Roy, S. J., Coates, J. C., et al. (2009). Shoot $\mathrm{Na}^{+}$exclusion and increased salinity tolerance engineered by cell typespecific alteration of $\mathrm{Na}^{+}$transport in Arabidopsis. Plant Cell 21, 2163-2178. doi: $10.1105 /$ tpc.108.064568

Munns, R., James, R. A., Xu, B., Athman, A., Conn, J. S., Jordans, C., et al. (2012). Wheat grain yield on saline soils is improved by an ancestral $\mathrm{Na}^{+}$transporter gene. Nat. Biotechnol. 30, 360-364. doi: 10.1038/nbt.2120

Munns, R., and Tester, M. (2008). Mechanisms of salinity tolerance. Ann. Rev. Plant Biol. 59, 651-681. doi: 10.1146/annurev.arplant.59.032607.092911

Platten, J. D., Cotsaftis, O., Berthomieu, P., Bohnert, H., Davenport, R. J., Fairbairn, D. J., et al. (2006). Nomenclature for HKT transporters, key determinants of plant salinity tolerance. Trends Plant Sci. 11, 372-374. doi: 10.1016/j.tplants.2006.06.001

Plett, D., Safwat, G., Gilliham, M., Moller, I. S., Roy, S., Shirley, N., et al. (2010). Improved salinity tolerance of rice through cell type-specific expression of AtHKT1;1. PLoS ONE 5:e12571. doi: 10.1371/journal.pone.0012571

Ren, Z. H., Gao, J. P., Li, L. G., Cai, X. L., Huang, W., Chao, D. Y., et al. (2005). A rice quantitative trait locus for salt tolerance encodes a sodium transporter. Nat. Genet. 37, 1141-1146. doi: 10.1038/ng1643

Rodriguez-Navarro, A. (2000). Potassium transport in fungi and plants. Biochim. Biophys. Acta 1469, 1-30. doi: 10.1016/S0304-4157(99)00013-1

Rubio, F., Gassmann, W., and Schroeder, J. I. (1995). Sodium-driven potassium uptake by the plant potassium transporter HKT1 and mutations conferring salt tolerance. Science 270, 1660-1663. doi: 10.1126/science.270.5242. 1660

Rubio, F., Schwarz, M., Gassmann, W., and Schroeder, J. I. (1999). Genetic selection of mutations in the high affinity $\mathrm{K}^{+}$transporter HKT1 that define functions of a loop site for reduced $\mathrm{Na}^{+}$permeability and increased $\mathrm{Na}^{+}$tolerance. J. Biol. Chem. 274, 6839-6847. doi: 10.1074/jbc.274.11.6839
Rus, A., Lee, B. H., Munoz-Mayor, A., Sharkhuu, A., Miura, K., Zhu, J. K., et al. (2004). AtHKT1 facilitates $\mathrm{Na}^{+}$homeostasis and $\mathrm{K}^{+}$nutrition in planta. Plant Physiol. 136, 2500-2511. doi: 10.1104/pp.104.042234

Rus, A., Yokoi, S., Sharkhuu, A., Reddy, M., Lee, B. H., Matsumoto, T. K., et al. (2001). AtHKT1 is a salt tolerance determinant that controls $\mathrm{Na}^{+}$ entry into plant roots. Proc. Natl. Acad. Sci. U.S.A. 98, 14150-14155. doi: 10.1073/pnas.241501798

Rus, A. M., Bressan, R. A., and Hasegawa, P. M. (2005). Unraveling salt tolerance in crops. Nat. Genet. 37, 1029-1030. doi: 10.1038/ng1005-1029

Schachtman, D. P., and Schroeder, J. I. (1994). Structure and transport mechanism of a high-affinity potassium uptake transporter from higher-plants. Nature 370, 655-658. doi: 10.1038/370655a0

Su, H., Balderas, E., Vera-Estrella, R., Golldack, D., Quigley, F., Zhao, C. S., et al. (2003). Expression of the cation transporter McHKT1 in a halophyte. Plant Mol. Biol. 52, 967-980. doi: 10.1023/A:1025445612244

Sunarpi, Horie, T., Motoda, J., Kubo, M., Yang, H., Yoda, K., et al. (2005). Enhanced salt tolerance mediated by AtHKT1 transporter-induced $\mathrm{Na}^{+}$unloading from xylem vessels to xylem parenchyma cells. Plant J. 44, 928-938. doi: 10.1111/j.1365-313X.2005.02595.x

Takahashi, R., Liu, S., and Takano, T. (2007). Cloning and functional comparison of a high-affinity $\mathrm{K}^{+}$transporter gene PhaHKT1 of salt-tolerant and salt-sensitive reed plants. J. Exp. Bot. 58, 4387-4395. doi: 10.1093/jxb/erm306

Tholema, N., Bakker, E. P., Bakker, E. P., Suzuki, A., and Nakamura, T. (1999). Change to alanine of one out of four selectivity filter glycines in KtrB causes a two orders of magnitude decrease in the affinities for both $\mathrm{K}^{+}$and $\mathrm{Na}^{+}$of the $\mathrm{Na}^{+}$dependent $\mathrm{K}^{+}$uptake system KtrAB from Vibrio alginolyticus. FEBS Lett. 450, 217-220. doi: 10.1016/S0014-5793(99)00504-9

Uozumi, N., Kim, E. J., Rubio, F., Yamaguchi, T., Muto, S., Tsuboi, A., et al. (2000). The Arabidopsis HKT1 gene homolog mediates inward $\mathrm{Na}^{+}$currents in Xenopus laevis oocytes and $\mathrm{Na}^{+}$uptake in Saccharomyces cerevisiae. Plant Physiol. 122, 1249-1259. doi: 10.1104/pp.122.4.1249

Yao, X., Horie, T., Xue, A. W., Leung, H. Y., Katsuhara, M., Brodsky, D. E., et al. (2010). Differential sodium and potassium transport selectivities of the rice OsHKT2;1 and OsHKT2;2 transporters in plant cells. Plant Physiol. 152, 341-355. doi: 10.1104/pp.109.145722

Conflict of Interest Statement: The authors declare that the research was conducted in the absence of any commercial or financial relationships that could be construed as a potential conflict of interest.

Received: 28 May 2014; accepted: 15 October 2014; published online: 04 November 2014.

Citation: Almeida PMF, de Boer G-J and de Boer AH (2014) Assessment of natural variation in the first pore domain of the tomato HKT1;2 transporter and characterization of mutated versions of SlHKT1;2 expressed in Xenopus laevis oocytes and via complementation of the salt sensitive athkt1;1 mutant. Front. Plant Sci. 5:600. doi: $10.3389 /$ fpls.2014.00600

This article was submitted to Plant Physiology, a section of the journal Frontiers in Plant Science.

Copyright (c) 2014 Almeida, de Boer and de Boer. This is an open-access article distributed under the terms of the Creative Commons Attribution License (CC BY). The use, distribution or reproduction in other forums is permitted, provided the original author(s) or licensor are credited and that the original publication in this journal is cited, in accordance with accepted academic practice. No use, distribution or reproduction is permitted which does not comply with these terms. 